\title{
A Multichannel Reflectometer for edge Density Profile Measurements at the ICRF Antenna in ASDEX Upgrade
}

\author{
O. Tudisco*, A. Silva ${ }^{\dagger}$, S. Ceccuzzi**, O. D’Arcangelo*, G. Rocchi*, \\ H. Fuenfgelder ${ }^{\ddagger}$, V. Bobkov ${ }^{\ddagger}$, R. Cavazzana ${ }^{\S}$, G.D. Conway ${ }^{\ddagger}$, J. Friesen ${ }^{\ddagger}$, \\ B. Gonçalves ${ }^{\dagger}$, A. Mancini*, L. Meneses ${ }^{\dagger}$, J.M. Noterdaeme ${ }^{\natural,+}$, G. Siegl ${ }^{\ddagger}$, \\ A. Simonetto ${ }^{\|}$, N. Tsujii ${ }^{\ddagger}$, A.A. Tuccillo*, T. Vierle ${ }^{\ddagger}$ I. Zammuto ${ }^{\ddagger}$, ASDEX \\ Upgrade Team ${ }^{\ddagger}$ and FTU Team* \\ *Associazione EURATOM-ENEA, C.R. Frascati, Via E. Fermi 45, 00044 Frascati, Italy \\ ${ }^{\dagger}$ Associação Euratom/IST, Instituto de Plasmas e Fusão Nuclear, Instituto Superior Técnico, Universidade \\ Técnica de Lisboa, 1049-001 Lisboa, Portugal \\ ${ }^{* *}$ Roma Tre University, Dept. of Engineering, Via V. Volterra 62, 00146, Roma, Italy \\ ${ }^{\ddagger}$ Max-Planck Institute für Plasmaphysik, EURATOM-Association IPP, D-85748 Garching, Germany \\ ${ }^{\S}$ Consorzio RFX, Euratom-ENEA Association, Padova, Italy \\ IUGent, Ghent, Belgium \\ ${ }$ CNR, Istituto di Fisica del Plasma, Associazione EURATOM-ENEA, Via R. Cozzi 53, 20125 Milano, Italy
}

\begin{abstract}
A multichannel reflectometer will be built for the new three-straps ICRF antenna of ASDEX Upgrade (AUG), to study the density behavior in front of it. Ten different accesses to the plasma are available for the three reflectometer channels that can be interchanged without breaking the machine vacuum. Frequency is scanned from $40 \mathrm{GHz}$ to $68 \mathrm{GHz}$, in $10 \mu s$, which corresponds to a cut-off density ranging from $10^{18} \div 10^{19} \mathrm{~m}^{-3}$ in the Right cut-off of the X-mode propagation, for standard toroidal magnetic field values of AUG.
\end{abstract}

Keywords: Reflectometry, plasma edge density, ICRF antenna, coupling PACS: $07.60 . \mathrm{Hv}$, 06.30.Dr, 52.35.-g, 52.50.Qt

\section{INTRODUCTION}

In order to minimize the electric field component parallel to the plasma magnetic field lines, two new antennas with three straps will be installed in ASDEX Upgrade (AUG). The parallel electric fields are expected to be reduced significantly [1] with an improvement of the impurity influx problem observed during ICRH. The antennas project is the result of a collaboration between IPP, ENEA and ASIPP (Hefei, China) laboratories and is foreseen to be installed in AUG in mid of 2014. Two of the present ICRF generators of AUG antennas will be used for the new ones, connected them in parallel, one will feed the two central straps and the other one the four external straps. To avoid impedance mismatching, a perfect geometric symmetry of the two antennas is adopted.

The behavior of the density in front of the antenna is a critical issue for the antenna-plasma coupling and, as it is influenced by ICRF electric field, its profile must be measured with a good time and space resolution. The reflectometry has been demonstrated to be an appropriate non-invasive method for plasma density measurements [2, 3] also for Scrape off Layer (SOL) density having several advantages, in terms of time and spatial resolution, with respect to the Langmuir probe diagnostic. Among the various reflectometer techniques, fast scanning reflectometers have been and are routinely used in many tokamaks for SOL $[4,5,6]$ and core $[3,7]$ density profile measurement. This system will be adopted for the new AUG antenna with a scan duration of $10 \mu \mathrm{s}$ repeated every $15 \mu \mathrm{s}$. Scanning duration is fast enough to avoid any phase decorrelation due to fluctuations.

One of the two antennas will be instrumented with a reflectometer capable of measuring the density in 3 different positions at the same time. The other antenna will be equipped with dummies waveguide components to keep the layout symmetry of the two antennas. Ten different locations have been identified to access the plasma and all will be equipped with pairs of waveguide for the reflectometer (bistatic antenna pairs). Due to reduced port room availability only 7 of these 10 accesses will be connected to the vacuum flange and can be fed without opening AUG vacuum. The other accesses, if required, can be connected in place of the other, only during a shut-down of the machine. Only 
TABLE 1. Frequency in GHz of the R-cutoff for various density and magnetic field. In bold are shown values covered by the reflectometer

\begin{tabular}{lcccccc}
\hline & \multicolumn{6}{c}{ Magnetic field at $R_{o}(\mathrm{~T})$} \\
Density $\left(10^{18} m^{-3}\right)$ & $\mathbf{1 . 5 0}$ & $\mathbf{1 . 7 5}$ & $\mathbf{2 . 0 0}$ & $\mathbf{2 . 2 5}$ & $\mathbf{2 . 5 0}$ & $\mathbf{2 . 7 0}$ \\
\hline 0.00 & 32.2 & 37.6 & $\mathbf{4 3 . 0}$ & $\mathbf{4 8 . 3}$ & $\mathbf{5 3 . 7}$ & $\mathbf{5 8 . 0}$ \\
1.00 & 34.6 & 39.6 & $\mathbf{4 4 . 8}$ & $\mathbf{5 0 . 0}$ & $\mathbf{5 5 . 2}$ & $\mathbf{5 9 . 4}$ \\
2.00 & 36.6 & $\mathbf{4 1 . 5}$ & $\mathbf{4 6 . 4}$ & $\mathbf{5 1 . 5}$ & $\mathbf{5 6 . 6}$ & $\mathbf{6 0 . 7}$ \\
3.00 & 38.5 & $\mathbf{4 3 . 5}$ & $\mathbf{4 8 . 0}$ & $\mathbf{5 2 . 9}$ & $\mathbf{5 7 . 9}$ & $\mathbf{6 1 . 9}$ \\
4.00 & $\mathbf{4 0 . 2}$ & $\mathbf{4 4 . 8}$ & $\mathbf{4 9 . 5}$ & $\mathbf{5 4 . 3}$ & $\mathbf{5 9 . 2}$ & $\mathbf{6 3 . 1}$ \\
5.00 & $\mathbf{4 1 . 9}$ & $\mathbf{4 6 . 3}$ & $\mathbf{5 0 . 9}$ & $\mathbf{5 5 . 6}$ & $\mathbf{6 0 . 4}$ & $\mathbf{6 4 . 3}$ \\
6.00 & $\mathbf{4 3 . 4}$ & $\mathbf{4 7 . 7}$ & $\mathbf{5 2 . 2}$ & $\mathbf{5 6 . 9}$ & $\mathbf{6 1 . 6}$ & $\mathbf{6 5 . 4}$ \\
7.00 & $\mathbf{4 4 . 8}$ & $\mathbf{4 9 . 1}$ & $\mathbf{5 3 . 5}$ & $\mathbf{5 8 . 1}$ & $\mathbf{6 2 . 7}$ & $\mathbf{6 6 . 5}$ \\
8.00 & $\mathbf{4 6 . 2}$ & $\mathbf{5 0 . 4}$ & $\mathbf{5 4 . 8}$ & $\mathbf{5 9 . 2}$ & $\mathbf{6 3 . 8}$ & $\mathbf{6 7 . 6}$ \\
9.00 & $\mathbf{4 7 . 5}$ & $\mathbf{5 1 . 7}$ & $\mathbf{5 5 . 9}$ & $\mathbf{6 0 . 4}$ & $\mathbf{6 4 . 9}$ & 68.6 \\
10.00 & $\mathbf{4 8 . 8}$ & $\mathbf{5 2 . 9}$ & $\mathbf{5 7 . 1}$ & $\mathbf{6 1 . 5}$ & $\mathbf{6 5 . 9}$ & 69.6 \\
\hline
\end{tabular}

three of the seven accesses will be instrumented with reflectometer at the same time. The reflectometer will be built in collaboration with IST of Lisbon.

\section{THE REFLECTOMETERS}

Frequency range. The expected range of density in front of the antenna is quite low $\left(<10^{19} \mathrm{~m}^{-3}\right)$, and right cut-off of X-mode propagation must be used. The required frequency range can be computed considering the operational space of AUG for typical discharges with ICRF. The magnetic field ranges from $1.5 T$ to $2.7 T$ at the plasma center $\left(R_{O}=1.65 \mathrm{~m}\right)$, and correspondingly at the antenna radius $(R=2.15 \mathrm{~m})$ ranges from $1.15 T$ to $2.07 T$. The corresponding frequency of the R-cutoff ranges from 32 to $70 \mathrm{GHz}$, as listed in table 1 . Actually, due to the poloidal extension of the ICRF antenna, the magnetic field at the antenna is not the same, and cutoff density also depends on the poloidal position of the reflectometer antenna. Frequency of the reflectometer, however, has been optimized for the plasma equatorial plane. The range of frequency of table 1 spans over $\mathrm{Q}, \mathrm{U}$ and $\mathrm{V}$ bands and to avoid using two oscillators a compromise has been obtained using a single custom oscillator from $40 \mathrm{GHz}$ to $68 \mathrm{GHz}$, in the U band (40-60 GHz) and part of the $\mathrm{V}$ band ( $50-75 \mathrm{GHz}$ ), covering most of the operational space. In table 1 the covered values are shown in bold. Standard waveguide WR19, in fundamental mode for U band and slightly oversized for V band, will be used to rely the power to and from the plasma.

Accesses to the plasma and antenna coupling. ICRF antennas are protected from plasma particle flux by a Faraday Shield (FS) made by set of parallel TZM rods, with $15 \mathrm{~mm}$ diameter at a distance of $22 \mathrm{~mm}$ aligned with magnetic field line for an average AUG discharge (about $12^{\circ}$ respect to the equatorial plane). The gap between two rods is 7 $\mathrm{mm}$ only. Two of ICRF antenna corners cannot be completely covered by the rods and some room is available for two pyramidal horns. In fig 1a a front view of the ICRF antenna is shown. The 10 accesses are identified by a progressive number (0-9) as shown in the picture. The detailed view of the lower left corner (access n.8) is shown in fig 1b, where the small hole between the rods and the frame is equipped with two horns for the reflectometry. The horns $(15 \mathrm{dBi})$ are oriented perpendicularly to the plasma surface and inclined compatibly with the surrounding structures, in order to maximize their coupling in presence of a reflecting surface that moves from $2 \mathrm{~cm}$ to $10 \mathrm{~cm}$ far from them. Analogues access is used at the top-right corner (access n.1). In order to have a comparison of the density inside the antenna frame and just outside it two accesses are foreseen at the same poloidal position just aside the antenna frame (accesses n. 0 and 9). Due to the limitation of the $7 \mathrm{~mm}$ gap between the rods in the accesses at the central part of the ICRF antenna, a detailed analysis has been done on several layout using a full wave code (CST Microwave Studio). As a first step horn antennas, elongated in the H-plane and shifted in the E-plane in between the rods of the Faraday Shield, have been considered, also considering the possibility of cutting part of the rods. Having evaluated that the mechanic impacts on the rods were not acceptable, it has been decided to use truncated oversized waveguides instead of the horns. Furthermore, to avoid possible sparks, the waveguide position has been retracted $5 \mathrm{~mm}$, behind the rods center. The coupling coefficient and the phase variation as a function of the reflecting cut-off position, have been computed 

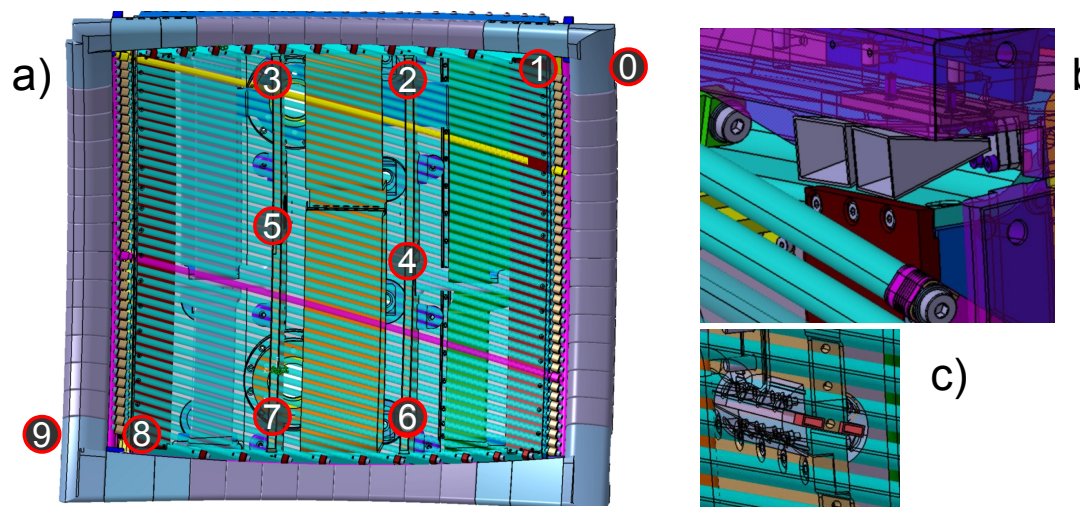

b)

FIGURE 1. a) Front view of the ICRF antenna. Accesses to the plasma are highlighted by red circles. b) detail of the upper right corner of the antenna, with the two horns for reflectometry. c) details of central accesses with two truncated WR42 waveguides.

$40 \mathrm{GHz}$ :
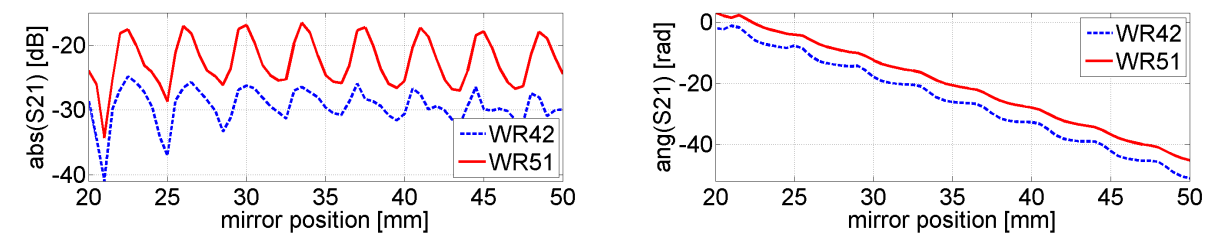

$60 \mathrm{GHz}:$
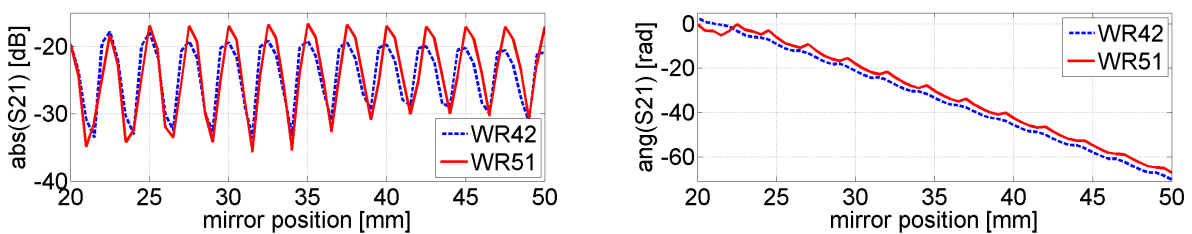

FIGURE 2. Coupling coefficient (S12) and phase variation as function of the position of the reflecting mirror (cut-off layer) for two frequencies and two WGs.

for two waveguides (WR42 and WR51) and two frequencies as shown in fig 2. Modulations observed on the coupling coefficients are due to multi reflections on metallic structure and cut-off layer which produce a relevant interference pattern that distorts both coupling and phase. Coupling oscillates between 20 and $35 \mathrm{~dB}$, while phase distortion, i.e. the discrepancy from the expected straight line, is of the order of $\pi / 2$ that correspond to a position indetermination of about $2 \mathrm{~mm}$. From an accurate analysis of the several metallic component effects, it turns out that multi reflections are between rods and reflecting mirror, in fact the interference reduces moving the mirror far from the horns and it is still present if the WG edge is set at the middle plane of the FS rods. Actually, this is probably a pessimistic result as TZM resistivity has been neglected and mirror has been considered perfectly flat. Density fluctuation will reduce the coefficient of the first reflection but will reduce the multi reflection effect furthermore. As no large differences in the coupling has been observed between WR42 (inner size $10.67 \times 4.33 \mathrm{~mm}$ ) and WR51 (inner size $12.95 \times 6.48 \mathrm{~mm}$ ), for assembling reasons the WR42 has been adopted. The effect of $10^{\circ}$ tilting of the reflecting mirror has also been computed and its effect remains within $3 \mathrm{~dB}$, due to the large antenna pattern of the truncated WG.

Power balance. The expected power at the detector can be computed, on the basis of the previous calculations. The Reflectometer oscillators will be located very close to the vacuum window in order to minimize the length of the waveguide. The present drawings shows that about $10 \mathrm{~m}$ of WG would be enough to cover the distance to and from the plasma. The attenuation of the WR19 waveguide including E and H curves, measured using a network analyzer available at the CNR institute of Milan, has been found to be about $-1.5 \mathrm{~dB} / \mathrm{m}$. Hence the insertion loss of the WG will be about $-15 \mathrm{~dB}$. It has been estimated that this attenuation is tolerable and the use of oversized WG can be avoided. Vacuum windows are made by $0.1 \mathrm{~mm}$ thick MICA disks. Same material will be used for DC break, inside and outside 


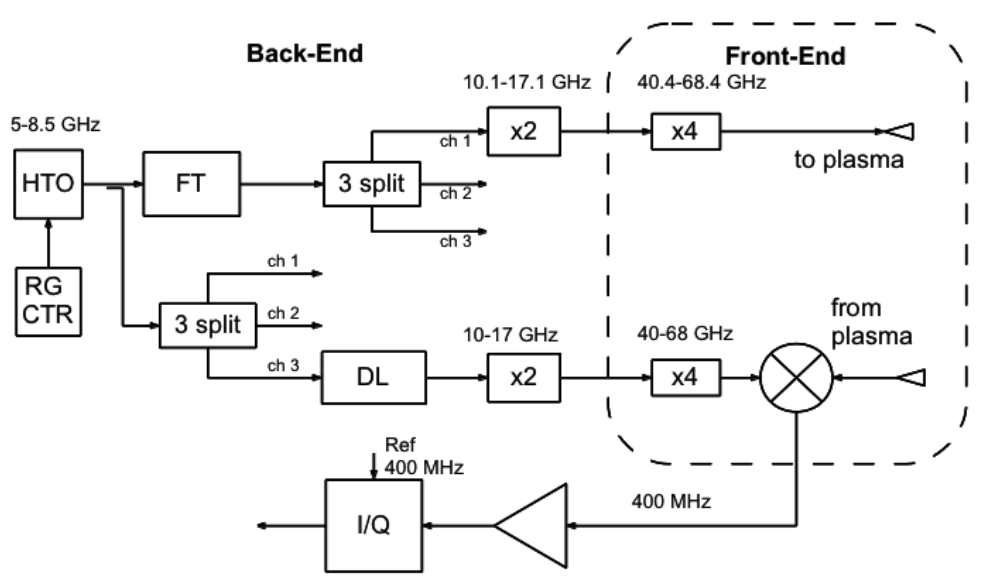

FIGURE 3. Layout of the reflectometer. HTO:Hyperabrupt Tuned Oscillator, RG CTR: Ramp Generator controller, FT : Frequency translator, DL : Delay Line.

the machine vacuum. The measured insertion loss of the MICA is almost negligible, but an attenuation of $-0.5 \mathrm{~dB}$ for each MICA insertion is considered. In the following the nominal losses of some relevant components are also listed.

$\begin{array}{lrl}\text { Oscillator output power } & 13 \mathrm{dBm} \\ \text { Vacuum Window double pass } & -1 \mathrm{~dB} \\ \text { Antenna coupling } & -35 \mathrm{~dB} \\ \text { Mixer conversion loss } & -9 \mathrm{~dB} \\ \text { Minimum detectable power } & -60 \mathrm{dBm}\end{array}$

$10 \mathrm{~m}$ of WR19 attenuation $\quad-15 \quad \mathrm{~dB}$

4 DC break $\quad-2 \quad d B$

WG isolator $\quad-2 \mathrm{~dB}$

Signal level at mixer $\quad-52 \mathrm{dBm}$

Using a pessimistic value for the coupling of $-35 \mathrm{~dB}$, and assuming a $13 \mathrm{dBm}$ source we get that the power at the mixer is about $-52 \mathrm{dBm}$, slightly higher that the minimum detectable value $(-60 \mathrm{dBm})$. In channels with horns (ch $0,1,8$ and 9 of fig 1), where the coupling is expected between $-20 \mathrm{~dB}$ and $-15 \mathrm{~dB}$, the power level will be much higher.

The electronic. The layout of the reflectometer electronic is shown in fig 3. It has been proposed and will be realized by IST of Lisbon. The scheme is similar to the other reflectometer already present on AUG [3] and to the one present on JET [7] for the core plasma density measurements. It is made by two main parts : the back end and front end. The front end contains the high frequency components, and includes the last frequency multiplication and detectors. This part will be located close to AUG port to reduce the WG length. The back end is the IF part of the system including the oscillators, controls and I/Q detections. The two blocks will be connected by low loss coaxial cable, and can be located farther from the tokamak. A frequency ramp $(5-8.5 \mathrm{GHz})$ is made by an externally controlled HTO in $10 \mu s$ every $15 \mu \mathrm{s}$, which output is split in two parts for the two arms of the reflectometer. One of the two is shifted in frequency by a Frequency Translator. Both arms are then split in three parts for three reflectometers. After a frequency multiplication by 2 and by 4 the ramp from $40 \mathrm{GHz}$ to $68 \mathrm{GHz}$ is obtained. In this configuration the three reflectometers will frequency sweep together. Operational flexibility in the frequency sweeping is not foreseen. The expected output power will be $13 \mathrm{dBm}$ in each channel.

\section{REFERENCES}

1. V. Bobkov et al, in Proc. of the 24th IAEA Fusion Energy Conference, San Diego, USA, vol. IAEA-CN-197. Vienna: IAEA, 2012, pp. EX/P5-19.

2. G.R. Hanson, J. B. Wilgen et al, Rev. Sci. Instrum. 66, 863 (1995)

3. A. Silva et al, Rev. Sci. Instrum. 67, 4138 (1996)

4. F Clairet, C Bottereau, J M Chareau, M Paume and R Sabot, Plasma Phys. Control. Fusion 43, 429 (2001)

5. G. R. Hanson, J. B. Wilgen, C. Lau, Y. Lin, G. M. Wallace, and S. J. Wukitch, Rev. Sci. Instrum. 79, 10 F114 (2008)

6. J.B. Wilgen et al, Rev. Sci. Instrum. 77, 10E933 (2006)

7. L. Meneses, L. Cupido, A. Sirinelli, M. E. Manso, and JET-EFDS Contributors, Rev. Sci. Instrum. 79, 10F108 (2008) 